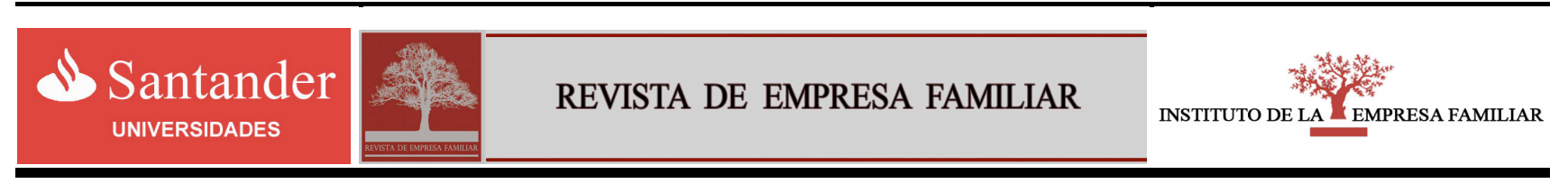

www.revistadeempresafamiliar.uma.es

\title{
Explorando las alianzas en la internacionalización de bodegas familiares españolas, argentinas y chilenas
}

\section{Exploring alliances in the internationalization of the family spanish, argentine and chilean wineries.}

\author{
Guadalupe Fuentes Lombardo ${ }^{\mathrm{a}, *} \cdot$ Manuel Carlos Vallejo Martos ${ }^{\mathrm{b}} \cdot$ Rubén Fernández Ortiz $^{\mathrm{c}}$ \\ a Departamento de Organización de Empresas, Marketing y Sociología. Universidad de Jaén, 23071 (Spain) \\ ${ }^{\mathrm{b}, \mathrm{c}}$ Departamento de Economía y Empresa. Universidad de La Rioja, 26004 (Spain)
}

\begin{tabular}{l} 
D A T O S A R T Í C U \\
\hline Historial: \\
Recibido 2010-07-20 \\
Aceptado 2011-02-22 \\
Palabras clave: \\
Alianzas estratégicas \\
Internacionalización \\
Empresa familiar \\
Sector vitivinícola \\
Códigos JEL: \\
M16
\end{tabular}

\section{A R T I C LE IN F O}

Article history:

Received 20 June 2010

Accepted 22 February 2011

\section{Keywords:}

Strategic Alliances

Internationalization

Family Firm

Wine Industry

JEL codes:

M16

\begin{abstract}
RES UMEN
Atendiendo a los distintos modos de entrada de una empresa en un mercado extranjero, el uso o no de alianzas estratégicas se podría ver condicionado por el carácter familiar de la empresa. En el caso concreto de las empresas familiares, dichas alianzas presentan una serie de ventajas e inconvenientes derivadas del peculiar carácter de estas empresas. Los resultados de este estudio cualitativo muestran los motivos que llevan a las empresas familiares a realizar o no alianzas estratégicas en su proceso de internacionalización, así como los factores discriminantes que favorecen o dificultan su selección en empresas del sector vitivinícola español, argentino y chileno. El grado de control de la familia en el negocio influye sobre ambos aspectos.
\end{abstract}

\section{A B S T R A C T}

When examining the different ways in which companies penetrate foreign markets, the decision about using or not strategic alliances may be conditioned by the characteristics of the company. In the specific case of family firms, such alliances offer a series of advantages and disadvantages due to the special characteristics of these companies. The results of this qualitative study show the reasons that prompt family businesses to establish or not strategic alliances in their internationalisation processes, as well as the discriminating factors that favour or hinder the selection of such strategies in the Spanish, Argentine and Chilean vinicultural companies. The degree of family control in the business influences both aspects.

\footnotetext{
*Autor de contacto. Tel.: +34953212340.

Correoselectrónicos: gfuentes@ujaen.es,mvallejo@ujaen.es,ruben.fernandez@unirioja.es
} 


\section{Introducción, justificación y objetivos de investigación}

Los investigadores no han sido ajenos al estudio de las alianzas estratégicas en el proceso de internacionalización empresarial. Sin embargo, hasta el momento, son escasos los trabajos que se centren en el análisis de los acuerdos de cooperación en la empresa familiar (Máñez, 2000; Niemelä, 2004; Roessl, 2005; Claver et al., 2007; Fuentes y Fernández, 2010) como modo de entrada a mercados internacionales.

Atendiendo a lo anterior, este trabajo de investigación trata de realizar una aportación a la literatura sobre alianzas estratégicas en el proceso de internacionalización de la empresa familiar, comparando empresas del sector vitivinícola español, argentino y chileno.

En primer lugar, dado el peculiar carácter de las empresas familiares, sus características pueden afectar a la estrategia de internacionalización, en general, y al establecimiento y desarrollo de acuerdos de cooperación con otras empresas, en particular. En segundo lugar, es necesario profundizar en si las posibles diferencias culturales existentes entre los tres países pueden contribuir a que los resultados de los objetivos de investigación planteados difieran en cada uno de ellos.

En el estudio empírico de este trabajo participan empresas del sector vitivinícola de España, Argentina y Chile. Silva y López de Ávila (2007) señalan que estos tres países se sitúan entre los seis primeros a nivel mundial atendiendo al volumen de litros exportados. En este sentido, Castillo y Rodríguez (2009) aluden al hecho de que el descenso del consumo de vino en el mercado interior (refiriéndose al caso de España) ha obligado a productores a buscar nuevas vías de comercialización para sus vinos tal como la exportación, es decir, uno de los modos de entrada que utilizan las empresas en el desarrollo de la estrategia de internacionalización.

Así, los objetivos de este trabajo son, primero, conocer cuáles son los motivos que llevan a la empresa familiar española a establecer o no alianzas estratégicas en su proceso de internacionalización, en comparación con aquellas otras empresas familiares argentinas y chilenas $\mathrm{y}$, segundo, investigar los factores que favorecen o dificultan el uso de alianzas estratégicas como modo de entrada en mercados exteriores. Analizar ambas cuestiones se justifica por tres razones que comentamos a continuación.

En primer lugar, trabajos de investigación previos (Fernández y Arranz, 1999; Holtbrügge, 2004) revelan que la formación de alianzas provee de distintos tipos de recursos $y$ capacidades a los socios, que aumentan la probabilidad de éxito en el proceso de internacionalización e incluso la presentan como la mejor forma de iniciarlo.

En segundo lugar, entre los recursos de los que proveen las alianzas estratégicas, algunos autores (Varadarajam y Cunningham, 1995; Iyer, 2002; Glaister, 2004) señalan el acceso a recursos de tipo financiero, a determinada información, capacidades de gestión, tecnología y mercados. Todos ellos podrían estar menos presentes en la empresa familiar (Seijo y Menéndez, 2004; Fernández y Nieto, 2005).

Finalmente, las características que presentan estas empresas, fruto del solapamiento de dos sistemas distintos, familia y empresa (Sharma, 2008), podrían influir notablemente en el uso de alianzas estratégicas en su proceso de internacionalización y en el éxito alcanzado con las mismas.

Para alcanzar los objetivos propuestos en este estudio hacemos uso de la metodología cualitativa y de la técnica de investigación de la entrevista en profundidad. Ambas se consideran las más adecuadas cuando el investigador pretende profundizar en el porqué y el cómo de un determinado fenómeno social (Taylor y Bogdan, 1992); en nuestro caso, conocer las posibles motivaciones y los factores que favorecen o dificultan el uso de alianzas estratégicas en la expansión internacional de estas compañías.

El trabajo ha sido estructurado en cuatro apartados, incluido este primero de carácter introductorio. En el segundo se realiza una revisión de la literatura que analiza las alianzas 
estratégicas en el proceso de internacionalización de la empresa familiar. E1 tercero describe la metodología empleada y las fases de trabajo desarrolladas hasta alcanzar los resultados de investigación. Finalmente, el cuarto, está destinado a comentar las principales conclusiones que se desprenden del trabajo y a exponer las limitaciones del mismo.

\section{Razones que justifican el uso de alianzas estratégicas en la internacionalización de la empresas familiar}

Las vías fundamentales de una empresa para acceder al mercado exterior son dos: exportación e inversión directa en el extranjero. Ambos modos de entrada podrán realizarse de manera individual o mediante el empleo de alianzas estratégicas (Fuentes y Fernández, 2010).

Para diversos autores (Porter y Fuller, 1986; Contractor y Lorange, 1988; Varadarajam y Cunningham, 1995; Townsend, 2003) el uso de acuerdos de cooperación se justifica por alcanzar o mantener una ventaja competitiva, minimizar costes, proveer de recursos y capacidades a los socios, desarrollar nuevos productos, acceder a canales de distribución, acelerar el proceso de internacionalización, reducir y/o diversificar el riesgo de la empresa y por otras razones de mercado, sociales y políticas.

En el caso de que la empresa desarrolle un proceso de internacionalización empresarial, el uso de alianzas estratégicas se justificaría por razones similares. Así, principalmente, el uso de acuerdos de cooperación en la expansión internacional de la empresa se explica por compartir recursos entre las empresas socio para mejorar la posición competitiva de ambas en el mercado de destino, por compartir riesgos y minimizar los costes que supone desarrollar la estrategia internacional, así como, por adquirir más información y un mayor aprendizaje que pueden ser muy útiles para acelerar la expansión internacional de la empresa.

Entre los trabajos que han analizado las alianzas estratégicas en el proceso de internacionalización de las empresas familiares
(Swinth y Vinton, 1993; Gallo y Cappuyns, 1999; Simon y Hitt, 2003; Ariño, 2005; Claver et al., 2007; Fuentes y Fernández, 2010), Gallo et al. (2002) consideran el modo en el que el carácter de estas empresas influye en las distintas fases del proceso de formación y desarrollo de las alianzas. En concreto, estos autores se refieren a determinadas diferencias provenientes de las características culturales de la empresa familiar, de sus procesos de dirección, del ciclo generacional en el que se encuentre inmersa y el nivel de uso de mecanismos de control. Simon y Hitt (2003) señalan cómo la mayoría de empresas familiares no suelen disponer de determinados recursos que son necesarios para competir eficazmente. Por ello, deben desarrollar sus capacidades o acceder a esos recursos, destacando las alianzas estratégicas como una de las alternativas para su obtención por permitir el acceso al aprendizaje de nuevas capacidades.

Atendiendo a los distintos tipos de alianzas estratégicas, Swinth y Vinton (1993) analizan las joint ventures internacionales entre empresas familiares y no familiares y argumentan como la probabilidad de éxito de una joint venture internacional aumenta cuando los socios son empresas familiares. La causa de lo anterior es el hecho de compartir determinados objetivos y valores. Así, comparten una visión a largo plazo, sistemas de gestión basados en la confianza, compromiso y lealtad, al mismo tiempo que otorgan una mayor importancia a la posibilidad de realizar cambios culturales, animando a los miembros de la familia a viajar e intercambiar puestos de trabajo entre las empresas que forman la joint venture.

De la revisión de la literatura realizada se desprende que las alianzas estratégicas permiten a las empresas familiares mejorar su posición competitiva e incrementar sus recursos y capacidades para desarrollar la estrategia de internacionalización de una forma más rápida y con mayores garantías de éxito. En este sentido, pueden convertirse en una alternativa para garantizar beneficios a largo plazo, lo que supondría asegurar la continuidad del negocio en la siguiente generación familiar. 


\subsection{Influencia de las fortalezas y debilidades de la empresa familiar en la elección y formación de alianzas estratégicas}

El conjunto de fortalezas que caracterizan a la empresa familiar (Ibrahim y Ellis, 1994; Kets de Vries, 1996; Poutziouris, 2001) presentan aspectos muy ventajosos para la formación $y$ éxito de las alianzas. La flexibilidad y rapidez en la toma de decisiones, que caracterizan a estas empresas, pueden afectar positivamente a la formación de alianzas (Gallo et al., 2002). Otra de sus fortalezas, las convicciones, valores y visión compartida (Tagiuri y Davis, 1996; Denison et al., 2004), podría favorecer la formación de alianzas estratégicas con otras empresas familiares que compartan su cultura organizativa (Swinth y Vinton, 1993). Además, la propiedad en manos de la familia (Kets de Vries, 1993; Ginebra, 1997), su orientación a largo plazo (Leach, 1993; Ibrahim y Ellis, 1994) y el mantenimiento de su identidad, pueden ser respetadas con la formación de estos acuerdos de cooperación (Terpstra y Simonin, 1993). Por último, los mayores niveles de compromiso y confianza que muestran estas empresas (Vallejo, 2008), caracterizadas por su fiabilidad y estabilidad $y$ por asumir con facilidad compromisos relacionados con su orientación a largo plazo (Leach, 1993; Kets de Vries, 1996), las convierte en candidatas idóneas para la formación de alianzas.

Considerando sus debilidades (Tagiuri y Davis, 1996; Ginebra, 1997; Poutziouris, 2001), la literatura nos muestra que en muchas ocasiones los socios exigen a estas empresas que su sucesión esté bien planificada, que la formación y experiencia de sus empleados sea buena y que no existan discordias familiares que puedan interferir en las relaciones de cooperación (Ariño, 2005). En esta línea, la rigidez y lentitud de cambios organizacionales, que quizás impliquen ausencia de un sistema profesionalizado, podrían afectar negativamente a la formación de alianzas (Gallo et al., 2002). Además, la autonomía en la toma de decisiones (Poza, 1995; Donckels y Lambrecht, 1999) más bien podría ser un obstáculo en tanto que las alianzas estratégicas implican compartir la toma de decisiones en determinadas actividades objeto del acuerdo. Finalmente, los problemas de financiación que muestran las empresas familiares (Friedman y Friedman, 1994; James, 1999) por no permitir, en ocasiones, la entrada de capital ajeno a la familia, podrían verse solventados con la creación de ciertos tipos de alianzas que no impliquen participación de capital del socio en la empresa familiar. A su vez, estas dificultades financieras pueden convertirse en el motivo que justifique el cierre de las alianzas, si en verdad la empresa desea llevar a cabo la estrategia de internacionalización y no dispone de recursos financieros suficientes para acometerla de manera individual (Glaister y Buckley; 1996; Glaister, 2004).

Finalmente, la falta de una clara definición estructural y el solapamiento de roles, o la falta de deseo de desvelar determinada información, podrían influir negativamente en la selección de estas empresas como socios en una alianza estratégica (Ariño, 2005).

Todos estos aspectos nos llevan a plantear los factores claves que se deberían considerar en la selección de alianzas estratégicas como modo de entrada en el proceso de internacionalización de la empresa familiar.

\subsection{Identificación de factores claves}

La revisión de la literatura sobre alianzas estratégicas en la empresa familiar y las peculiaridades que caracterizan a estas empresas nos llevan a plantear aquellos aspectos claves que podrían influir en la formación y desarrollo de acuerdos de cooperación en la estrategia internacional de estas compañías. Así, la elección de este modo de entrada podría verse condicionada por tres factores. Primero, que la alianza implique la participación o no de la empresa socio, en el capital de la empresa familiar; segundo, que la empresa socio tenga o no carácter familiar $\mathrm{y}$; finalmente, que la empresa presente o no algunas de las debilidades que caracterizan a la empresa familiar.

En primer lugar, las empresas familiares podrían rechazar realizar un acuerdo de cooperación que implicase participación en su 
capital, ya que ello supondría ceder parte del control e incluso poner en peligro el propio carácter familiar y la identidad del negocio. No obstante, cabe la posibilidad de que las empresas familiares participen en el capital social de las empresas del país de destino o de una nueva que se cree. Así, estas compañías podrían encontrar en esta alianza la ayuda financiera para desarrollar su estrategia de internacionalización.

En segundo lugar, si la empresa socio fuese un negocio no familiar, ambas podrían beneficiarse de los recursos tangibles e intangibles que las caracterizan.

Así, la empresa no familiar podría obtener los recursos intangibles (know how, experiencia e imagen, etc.) que constituyen fortalezas de las empresas de carácter familiar (Leach, 1993; Ginebra, 1997), y éstas, a su vez podrían obtener los recursos financieros y tecnológicos que podrían estar presentes, en mayor medida, en empresas no familiares (Chandler, 1990). Sin embargo, la mayoría de las alianzas estratégicas realizadas por empresas familiares suelen ser con otras empresas de su mismo carácter. Una explicación a este hecho puede encontrarse en la cultura de este tipo de empresas (Máñez, 2000; Roessl, 2005), pues existen valores, compromisos e incluso objetivos y estrategias compartidas que permiten favorecer tanto la formación como el buen desarrollo de las alianzas.

Finalmente, cuando una empresa familiar presenta debilidades derivadas de su peculiar carácter, difícilmente podrá formar alianzas estratégicas en su expansión internacional con otras empresas, pues estas últimas podrían no considerarlas como el socio más adecuado. Nos referimos a un relevo generacional del negocio no planificado, distintas formas de nepotismo que pueden dificultar la entrada de directivos externos profesionales, discordias familiares que afecten al buen desarrollo del negocio, falta de una estructura organizativa clara y el secretismo motivado por el no deseo de desvelar información de la empresa a terceros ajenos a la familia.

\section{Metodología de la investigación}

\subsection{Selección de empresas objeto de estudio}

Dentro de la fase de diseño del trabajo resulta de vital importancia la selección de la muestra de empresas que participan en el estudio. En relación a este aspecto, se han elegido empresas familiares pertenecientes al sector vitivinícola español, argentino y chileno que estuviesen desarrollando una estrategia internacional. El sector seleccionado es el vitivinícola, pues España ocupa el segundo puesto a nivel mundial ${ }^{1}$, después de Italia, atendiendo al volumen de litros exportados, y el tercer puesto atendiendo al valor de sus ventas en el exterior (Suárez-Ortega, 2003). En el caso de Argentina y Chile (que juntos se sitúan como quintos exportadores a nivel mundial por el volumen de litros exportados ${ }^{2}$ ), estos países están dentro de los denominados como países del Nuevo Mundo cuyos productos son fuertes competidores de los vinos de nuestro país a nivel internacional y, de ahí, la importancia de realizar estudios comparativos entre los tres países. En el caso de España, utilizamos para discriminar a las compañías que participan en este estudio la base de datos empresariales SABI, en el caso de las bodegas argentinas, esta discriminación fue realizada por directivos de la Fundación ProMendoza y en Chile, nos apoyamos de la información suministrada por el personal y directivos de La Dirección de Promoción de Exportaciones -ProChile-.

En el estudio empírico han participado un total de 75 empresas (36 bodegas españolas, 23 bodegas argentinas y 16 bodegas chilenas), en las cuales se han realizado 93 entrevistas, en su conjunto, a directivos y miembros del Consejo de Administración, familiares y no familiares, relacionados con la actividad internacional de la empresa. Estas entrevistas fueron realizadas durante el periodo de tiempo comprendido entre enero y abril de 2007, en el caso de España, entre septiembre y diciembre de 2008 , en el

\footnotetext{
Según los datos de 2009 publicados por la Organización Internacional de la Viña y del Vino (OIV).

Según los datos de 2009 publicados por la Organización Internacional de la Viña y del Vino (OIV).
} 
caso de Argentina y, finalmente, entre mayo y septiembre de 2009, en el caso de Chile. El número de entrevistas realizadas en cada país vino determinado a partir de la saturación de la información recogida en las entrevistas.

\subsection{Recogida de información}

La información utilizada para realizar el estudio empírico ha provenido de dos fuentes, fichas de empresas y discursos contenidos en las entrevistas. La ficha de cada empresa se elaboró con información secundaria que provenía de distintas bases de datos empresariales, publicaciones periódicas del sector, páginas web de las empresas, etc. Esta información se relacionaba con el carácter familiar o no de la compañía, con el desarrollo o no de una estrategia internacional, con la elección o no de alianzas estratégicas como modo de entrada a los mercados extranjeros, además de con otros aspectos relacionados con los modos de entrada a los mercados exteriores o el tamaño de la compañía. De otro lado, las cuestiones planteadas durante las entrevistas se relacionaban con las motivaciones para establecer o no alianzas estratégicas y los factores que habían favorecido o dificultado la elección o no de este modo de entrada. En este sentido, debemos señalar que estas cuestiones fueron depuradas tras la realización de cuatro entrevistas piloto, quedando definitivamente como siguen:

1. Motivaciones para usar acuerdos de cooperación en el desarrollo de su proceso de internacionalización, en lugar de realizarlo de manera individual.

2. Razones que ayudaron a poder establecer acuerdos de cooperación.

3. Razones que dificultaron en principio el establecimiento de acuerdos de cooperación.

4. Influencia del carácter familiar de su empresa en el establecimiento de los acuerdos de cooperación y en el éxito alcanzado con los mismos.

Todas las entrevistas fueron realizadas por los autores de este trabajo. Durante la realización de las mismas se optó por su grabación con el objetivo de realizar su posterior transcripción $\mathrm{y}$, de este modo, disponer de toda la información necesaria para realizar el análisis de los discursos sin que el entrevistador perdiese posibles detalles de la conversación.

\subsection{Análisis de la información}

La principal fuente de información utilizada en el estudio empírico de este trabajo es aquella que proviene de los discursos de las entrevistas realizadas. Para el análisis e interpretación de los discursos nos hemos basado fundamentalmente en los trabajos realizados por numerosos autores (Taylor y Bodgan, 1992; Verón, 1996 y Alonso, 1998) sobre el Análisis sociológico de los discursos. Así, Alonso (1998) señala que en el análisis de los discursos se trata de realizar la reconstrucción del sentido de los discursos en su situación de enunciación, encontrando un modelo de representación y comprensión del texto concreto en su contexto social $y$ en la historicidad de sus planteamientos, desde la reconstrucción de los intereses de los actores que están implicados en el discurso.

De este modo, la preparación de nuestro análisis comenzó por una trascripción literal de las entrevistas y una lectura (escucha) varias veces repetida de todas las entrevistas realizadas. Tras esta fase, se inició una reconstrucción personal de la información contenida en las mismas, en la que establecimos las relaciones entre las distintas categorías que son objeto de nuestro interés, en relación a los objetivos de investigación planteados en este trabajo. Una vez analizada la información por los autores, los resultados obtenidos se triangularon con los alcanzados por otros investigadores a los que se les proporcionaron los discursos de las entrevistas realizadas.

\section{Resultados de investigación}

\subsection{Motivaciones para usar o no alianzas estratégicas en el desarrollo del proceso de internacionalización}


En primer lugar, vamos a referirnos a aquellas empresas que han optado por desarrollar de manera individual su estrategia de internacionalización y que han sido más frecuentes en el caso español que en los casos de Argentina y Chile. De este modo, los argumentos que los directivos y propietarios de estas empresas nos dan para no desarrollar acuerdos de cooperación y la influencia que sobre lo anterior tiene el carácter familiar o no de la empresa, son:

- Prefieren conservar su autonomía y el control total de la toma de decisiones. En este sentido, las alianzas estratégicas que han podido realizar implicaban que los posibles socios participaran en el capital de la compañía y han sido rechazadas rotundamente por parte de sus propietarios, que anteponen una propiedad del negocio completamente familiar a los posibles beneficios que podrían obtenerse de estos acuerdos.

- Consideran favorable no abordar proyectos en la actualidad si no tienen los recursos suficientes y esperar a un futuro en el que estos recursos se hayan generado en la propia empresa, gracias a la autofinanciación de la compañía, antes que cooperar, sobre todo si esta cooperación implica ceder parte de la propiedad y control a la empresa socio.

- Los directivos familiares consideran que les beneficia realizar de manera individual y directa la venta de sus productos en el extranjero porque les es más rentable (no tienen que pagar comisiones a intermediarios). Además, ellos creen que son capaces de defender sus productos en el exterior de una manera más favorable y directa, pues impregnan a los clientes de una calidad e imagen de sus productos y empresa, que difícilmente podrían hacerlo terceros ajenos a la compañía.

- En el caso de que los acuerdos se hayan intentado plantear con otras empresas del sector y de la misma zona geográfica, han surgido numerosos conflictos y no se han obtenido buenos resultados por la competencia de los productos.

- Muestran una actitud reacia a establecer acuerdos de cooperación con otras compañías.
En muchas ocasiones esta mentalidad cerrada para formar alianzas es consecuencia del orgullo que los dueños sienten por su empresa que les lleva a querer comercializar sus productos y sus marcas para ser reconocidos en el mercado con una cierta exclusividad, pese a que directivos no familiares de la empresa no estén de acuerdo con esta filosofía.

- Consideran difícil encontrar una posible empresa socio cuya filosofía, intereses y objetivos sean compatibles con los de su propia compañía.

- En ocasiones, miembros de una misma familia deciden separarse y no cooperar entre ellos, pese a las ventajas, que son conscientes, que le podría ofrecer la cooperación en costes, volúmenes, etc. Las razones argumentadas han sido la timidez o el miedo a que ello pueda suponer conflictos dentro de la familia.

- Dentro de este apartado debemos destacar que mientras que las bodegas familiares españolas señalaban como otro motivo para desarrollar su internacionalización de manera individual el no estar dispuestos a cooperar con otras empresas porque ello implicaría desvelar información que los propietarios consideran relevante y confidencial de su familia y de su negocio, este argumento no se ha reflejado en los resultados obtenidos para las empresas argentinas ni chilenas.

En segundo lugar, los directivos de las empresas que sí han optado por el uso de alianzas estratégicas para el desarrollo de su estrategia de internacionalización, la mayoría de bodegas argentinas y chilenas, nos han argumentado las siguientes motivaciones para desarrollarlas:

- La necesidad de ampliar la oferta con los productos de otras empresas distribuidoras, siempre y cuando estos resulten ser complementarios e incluso impliquen la obtención de sinergias en costes de marketing, comercialización y distribución.

- La mayor información y la mejora en la toma de decisiones que se puede producir cuando se tengan distintos puntos de vista que pueden favorecer los resultados de la empresa. 
- En ocasiones, la formación de alianzas con otras empresas del mercado de destino es ineludible cuando se desea entrar en un determinado país, pues lo exigen las propias reglamentaciones o, aunque no sea así, directivos argentinos y chilenos consideran que el llegar a establecer una alianza con una bodega española, por ejemplo, podría ser la puerta de entrada y ayudarles enormemente a alcanzar un mercado más amplio tal y como es el caso de Europa.

- Algunas empresas desarrollan acuerdos con otras compañías de la zona para repartir los costes que supone la venta en el mercado exterior de sus productos. Aún así, debemos considerar que estos acuerdos y los socios que los forman son organizados por los propios importadores. Se trata de empresas cuyo volumen de ventas en el extranjero resulta ser tan pequeño que no les sería rentable despachar sus productos de manera individual.

A diferencia de las bodegas españolas y argentinas, en las chilenas se han observado una mayor cantidad de acuerdos de cooperación realizados con proveedores de distinta nacionalidad. Una de las razones que explican estos acuerdos de cooperación internacionales se relaciona con la escasez de empresas chilenas proveedoras del sector vitivinícola (maquinaria, envases de distintos materiales, corcho, etc.).

En relación al uso de otros acuerdos de cooperación (con distribuidores y/o competidores) los motivos dados por los directivos de bodegas chilenas suelen ser coincidentes a los de las bodegas españolas y argentinas, aumentar la cuota de mercado por compartir recursos, por ejemplo por ampliar la cartera de productos que se ofrece a clientes internacionales, $o$ adquirir un mayor conocimiento e información sobre los mercados a los cuales se dirigen.

\subsection{Razones que favorecen el establecimiento de acuerdos de cooperación}

De otro lado, los resultados del análisis de los discursos contenidos en las entrevistas nos muestran que las razones que han favorecido el establecimiento de estos acuerdos han sido:
- Compartir recursos con otras empresas, cediendo el saber hacer de la compañía, la elaboración de sus productos, recursos tecnológicos, servicios de marketing y de comercialización, y adquiriendo el saber hacer de un socio local del país de destino o los recursos financieros para poder desarrollar nuevos proyectos de manera conjunta, así como acceder a los productos elaborados por otras empresas para aumentar la gama de productos de la compañía.

- La incorporación de nuevas generaciones al negocio con una visión y actitudes menos conservadoras y más arriesgadas a las de la generación anterior. La mayor antigüedad y tradición de las bodegas españolas hace que las personas en las que recae la toma de decisiones muestren una mayor reticencia a la cesión de control que supone el establecimiento de una alianza estratégica, si las comparamos con las bodegas chilenas y argentinas cuyos propietarios, desde el preciso instante de su fundación, muestran actitudes más parecidas a empresas born globals. Sin embargo, estas diferencias en la disposición a ceder parte del control que supone una alianza estratégica entre bodegas españolas por un lado y argentinas y chilenas por otro, tiende a disminuir a medida que las nuevas generaciones familiares se ponen al frente de las bodegas españolas.

- La existencia de relaciones personales de amistad, profesionales y comerciales con otras empresas a lo largo del tiempo. En este sentido, las alianzas que establecen estas empresas surgen con otras empresas familiares, en la mayoría de los casos, o con empresas que pese a no ser familiares tienen entre sus directivos personas de total confianza y con las que también se han mantenido con anterioridad relaciones personales. En las compañías chilenas, que dada su localización geográfica y el tamaño de su mercado doméstico, se han visto más "obligadas" a competir de manera internacional desde su creación, estas relaciones eran más frecuentes.

- La existencia de objetivos y estrategias similares y compartidas con la empresa socio en los mercados en los que se desean introducir, así como, de una comunicación fluida e 
información precisa de los objetivos e intereses de cada socio en relación al acuerdo, tanto en el inicio como durante el desarrollo del mismo. Esta razón es más frecuente en los casos en los que la empresa socio es también una empresa familiar.

- La similitud de tamaño de las empresas que pretenden cooperar y de sus estructuras organizativas. Así, en el caso de las bodegas chilenas y argentinas se buscan distribuidores extranjeros que no cuenten en su cartera de productos con otros vinos de estos países, requisito de exclusividad menos señalado por los directivos de bodegas españolas.

Para el caso concreto de bodegas argentinas y chilenas, además de las razones que favorecen el uso de alianzas estratégicas señaladas anteriormente, también se observaron las siguientes:

- La posibilidad de compartir con distribuidores internacionales los gastos de marketing en los países de destino.

- El perfil del importador, pues la mayoría de las bodegas argentinas y chilenas consideran positivo que el distribuidor sea una empresa familiar que no tenga gran tamaño y que no se encuentre especializado en un tipo concreto de vino (precio bajo o precio alto), sino que posea una amplia variedad de productos dentro de su cartera.

- Las relaciones con otros agentes del sector, la asistencia a diversos actos (jornadas, congresos, etc.), los desplazamientos a los mercados en los que están presentes, e incluso a otros en los que desean entrar, son algunos más de los aspectos que favorecen la formación de acuerdos de cooperación con otras compañías, sobre todo en cuestiones relacionadas con la comercialización y distribución de sus productos.

Sólo en el caso de bodegas chilenas se añadió otro aspecto favorecedor de la formación de alianzas además de los señalados anteriormente. Así, para los directivos de estas compañías, favorece la realización de acuerdos de cooperación que las bodegas se encuentren situadas en distintos valles y elaboren vinos de distintas variedades (cepas) cuya filosofía del negocio, la imagen del producto y la estrategia empresarial sea similar.

\subsection{Razones que dificultan el establecimiento de acuerdos de cooperación}

Finalmente, los directivos de las bodegas de los tres países señalaron como aspectos que dificultaron la formación de alianzas estratégicas con otras empresas los siguientes:

- Los objetivos e intereses que cada uno de los socios persigue con la formación de la alianza no son compatibles, así como tampoco lo son las estructuras organizativas de ambas empresas. Esto suele ocurrir en mayor medida cuando la posible empresa socio no tiene carácter familiar.

- El intento de establecer acuerdos con otras empresas cuyos productos son competidores.

- La dificultad de encontrar una posible empresa socio en la que poder confiar, cuya filosofía e intereses no sean contradictorios con los de la compañía que, además, sea el socio más adecuado en el país que la bodega desea entrar y que se pueda llegar con él a un acuerdo en todos los aspectos que se deben considerar cuando se está negociando una alianza.

Uno de los factores que para las empresas familiares españolas dificultaba la formación de alianzas estratégicas era que el accionariado estuviese muy diluido, ya que para llegar a cualquier acuerdo, tal como podía ser establecer una alianza estratégica con otra empresa, se requería que un gran número de propietarios lo considerase conveniente. En el caso de las bodegas argentinas y chilenas este argumento no se ha observado debido, quizás, a la mayor concentración de capital que existe en estas compañías.

La mayoría de los directivos entrevistados consideran que difícilmente se pueden crear acuerdos de cooperación que impliquen el reparto de la propiedad de la empresa o compartir la toma de decisiones con terceros ajenos a la compañía porque ello implicaría una pérdida de identidad del negocio. Así, tan solo se han observado tres joint venture entre las empresas que han participado en el estudio y 
que fue necesario realizar para entrar en el mercado de EE.UU. por una bodega argentina y por dos españolas, sin embargo, encontramos una mayor disposición de los directivos argentinos y chilenos a establecer este tipo de alianzas que en el caso de los directivos españoles.

Aunque muchas bodegas chilenas consideran beneficiosa la realización de acuerdos de cooperación, una de las razones expuestas para no desarrollarlos fue no haber recibido ninguna oferta atractiva de cooperación con otra u otras empresas del sector. En este sentido, existe una clara diferencia con las bodegas españolas que sí recibían numerosas ofertas y con las bodegas argentinas, aunque estas últimas en menor cantidad que las anteriores.

En el caso de las bodegas chilenas, otro argumento esgrimido como dificultad para la formación de alianzas estratégicas fue la consideración poca ventajosa del logro de mayores economías de escala cuando la compañía disfruta de niveles de producción por encima de su umbral de rentabilidad, si para ello era necesario cooperar con otras empresas competidoras, dados los posibles inconvenientes que el acuerdo podría conllevar, esto es, conflicto de intereses o filosofías distintas de negocio. Así, en la mayoría de los casos la rentabilidad y la obtención de beneficios no han sido los objetivos prioritarios que han señalado los directivos familiares entrevistados puesto que, en ocasiones, lo que se persigue es alcanzar el status social que da la condición de ser "propietario y/o directivo de una bodega".

\section{Conclusiones generales}

Los resultados obtenidos en este trabajo de investigación muestran cómo el carácter familiar de las empresas del sector vitivinícola parece influir en el establecimiento de acuerdos de cooperación y éxito alcanzado con los mismos, en el desarrollo de la estrategia de internacionalización.

Así, en primer lugar, los propietarios de las bodegas familiares españolas, argentinas y chilenas no están dispuestos a realizar acuerdos de cooperación que impliquen la cesión de parte de la propiedad de la empresa a terceros ajenos a la familia, en la mayoría de los casos, por la posible pérdida de control en la toma de decisiones del negocio que podría conllevar esta decisión.

Sin embargo, existe una mayor posibilidad, a partir de la actitud no totalmente reacia de los directivos argentinos y chilenos, de poder realizar estos acuerdos. La explicación a lo anterior puede encontrarse en la menor disponibilidad de recursos financieros e intangibles (tecnológicos, organizativos, relacionales y humanos) con los que cuentan las bodegas argentinas y chilenas frente a las españolas.

En segundo lugar, cuando el acuerdo no implica cesión de la propiedad a un tercero, los socios que se buscan son preferentemente aquellos que tengan un tamaño empresarial similar y objetivos, intereses, cultura, etc.., parecidos a los de la compañía. Lo anterior suele ocurrir con mayor frecuencia cuando el posible socio es otra empresa familiar. Este resultado ha sido similar en los tres países, si bien, directivos argentinos y chilenos estarían dispuestos a realizar alianzas estratégicas con empresas que no posean todas las características anteriores en mayor medida que las empresas españolas.

En tercer lugar, un factor importante tiene que ver con las relaciones mantenidas anteriormente con otros agentes relacionados con el sector, pues ayudan a los directivos de estas compañías a formar acuerdos de cooperación.

Así, estas bodegas familiares buscan socios que les generen confianza, que conozcan por relaciones profesionales realizadas previamente y que gocen de un reconocido prestigio en el mercado nacional y/o internacional, conseguido por su imagen de seriedad (relacionada con el apellido de una familia). Si bien, son las empresas españolas las que dentro del sector y a nivel internacional pueden gozar de una mayor dotación de recursos relacionales, las empresas argentinas muestran mayor interés por crear y fomentar estas relaciones, incluso en mayor medida que las bodegas chilenas. 
Finalmente, la mayor concentración de la propiedad y dirección en las bodegas chilenas y argentinas, en comparación con las españolas, implica que la toma de decisiones relativa al establecimiento de acuerdos de cooperación con terceros sea más rápida y sencilla (en un sentido o en otro) que en las bodegas españolas, donde la mayor dispersión de la propiedad y dirección hace más difícil la adopción de cualquier tipo de acuerdo en general y, aún más, cuando se trata de estudiar la posible formación de una alianza estratégica con otra $\mathrm{u}$ otras empresas para desarrollar la estrategia internacional.

A modo de síntesis, este estudio expone similitudes en relación a los objetivos estudiados entre las bodegas familiares españolas, argentinas y chilenas, pero al mismo tiempo también refleja determinadas diferencias que se explican por las distintas culturas geográficas, la diferencia en dotación de recursos y la tradición y antigüedad que cada uno de los tres países posee en el sector.

Pese a la importancia de los resultados obtenidos en este trabajo y su contribución al estudio de las alianzas estratégicas en la internacionalización de las empresas familiares dada la escasez de literatura existente, en relación a los objetivos de investigación analizados, los mismos no pueden ser generalizados a otros países ni a otros sectores pues se necesitaría de un estudio empírico de carácter cuantitativo para poder llegar a la generalización de los mismos. Otra limitación que presenta este estudio es el haber realizado las entrevistas en distintos periodos de tiempo para cada uno de los países, de modo que la coyuntura económica de cada país, en cada momento del tiempo también ha podido influir en los resultados alcanzados.

\section{Bibliografía}

Alonso, L.E. (1998). La mirada cualitativa en sociología. Madrid: Fundamentos.

Ariño, A. (2005). Las alianzas estratégicas: una opción para potenciar el crecimiento de la empresa familiar. En M. Garrido y J.M. Furgado (Eds.), El Libro Blanco sobre patrimonio familiar, empresarial y profesional. Sus protocolos (pp. 631-675) Barcelona: Bosch, S.A.

Castillo, J.S. y Rodríguez, M. (2009). Determinantes de la evolución del mercado del vino en España. Distribución y Consumo, Noviembre-Diciembre, 70-89.

Chandler, A. (1990). Scale and scope. The dynamics of industrial capitalism. Cambridge, Mass: Harvard/Belknap.

Claver, E.; Rienda, L. y Quer, D. (2007). Incide el carácter familiar en el compromiso internacional de las empresas españolas, Investigaciones Europeas de Dirección y Economía de la Empresa, 13. 13-32.

Contractor, F.J. y Lorange, P. (1988). Why should firms cooperate? The strategy and economics basis for cooperative ventures. En F.J. Contractor y P. Lorange (Eds.), Cooperative strategies in international business (pp. 1-28). Toronto: Lexington, MA.

Denison, D., Lief, C. y Ward, J. (2004). Culture in family-owned enterprises: Recognizing and leveraging unique strengths. Family Business Review, 17(1), 61-70.

Donckels, R. y Lambrech, J. (1999). Are family businesses really different? What we know from Western European business research that could be applied to the reemergence of family-based. Enterprises in East Central Europe. Family Business Review, 12(2), 171-191.

Fernández, J.C. y Arranz, N. (1999). La cooperación entre empresas. Análisis y diseño. Madrid: ESIC, Escuela Superior de Gestión Comercial y Marketing.

Fernández, Z. y Nieto, M.J. (2005). Internationalization strategy of small and medium-sized family businesses: Some influential factors. Family Business Review, 18(1), 77-89.

Friedman, M. y Friedman, S. (1994). How to run a family business. Cincinnati: $\mathrm{OH}$ : Better way Books.

Fuentes, G. y Fernández, R. (2010): Strategic Alliances in the Internationalization of Family Fims: An Exploratory Study on the Spanish 
Wine Industry. Advances in Management, 3(6), 45-54.

Gallo, M.A. y Cappuyns, K. (1999). La internacionalización de la empresa familiar. El papel de las alianzas estratégicas. Iniciativa Emprendedora y Empresa Familiar, 16, 38-44.

Gallo, M.A., Ariño, A., Máñez, I. y Cappuyns, K. (2002). Internacionalización vía alianzas estratégicas en la empresa familiar. Research paper 447. Barcelona (Spain): IESE.

Ginebra, J. (1997). Las empresas familiares. Mexico: Panorama.

Glaister, K.W. (2004). The rationale for international equity joint ventures. European Management Journal, 22(5), 493-507.

Glaister, K.W. y Buckley, P.J. (1996). Strategic motives for international alliance formation. Journal of Management Studies, 33(3), 301332.

Holtbrügge, D. (2004). Management of international strategic business cooperation: Situational conditions, performance criteria, and success factors. Thunderbird International Business Review, 46(3), 255-274.

Ibrahim, A. y Ellis, W. (1994). Family business management. Concepts and practice. Dubuque: Kendall/Hunt Publishing Company.

Iyer, K. (2002). Learning in strategic alliances: An evolutionary perspective. Academy of Marketing Science Review, 10, 1-14.

James JR., H.S. (1999). What can the family contribute to business? Examining contractual relationships. Family Business Review, 12(1), 61-72.

Kets de Vries, M. (1993). Lo bueno y lo malo de las empresas de titularidad familiar. Harvard Deusto Business Review, 4, 32-44.

Kets de Vries, M. (1996). Family Business: Human Dilemmas in the Family Firm. London: Thomson Business Press.

Leach, P. (1993). La empresa familiar. Barcelona: Granica.

Máñez Testor, I. (2000). Continuidad y crecimiento de la empresa familiar. La vía de la cooperación y el cierre de alianzas estratégicas. Valencia (Spain): OPVI.

Niemelä, T. (2004). Interfirm cooperation capability in the context of networking family firms: The role of power. Family Business Review, 17(4), 319-330.

Organización internacional de la viña y del vino (OIV) (2009). Balance de la OIV sobre la situación vitivinícola mundial en 2009. Recuperado el 24 de febrero de 2011, del sitio Web de la Organización Internacional de la Viña y el Vino:

http://news.reseauconcept.net/images/oiv_es/Cli ent/Communique_Stats_Tbilissi_ES.pdf

Porter, M.E., y Fuller, M. (1986). Coalitions and global strategy. En M.E. Porter (Ed.), Competition in global industries (pp. 315-343). Boston: Harvard Business School Press.

Poutziouris, P.Z. (2001). The views of family companies on venture capital: empirical evidence from the UK small to medium-size enterprising economy. Family Business Review, 14(3), 277-291.

Poza, E. (1995). A la sombra del roble: La empresa privada familiar y su continuidad. Ohio: Editorial Universitaria para la Empresa Familiar.

Roess1, D. (2005). Family businesses and interfirm cooperation. Family Business Review, 18(3), 203-214.

Seijo Marcos, E. y Menéndez Requejo, S. (2004). Internacionalización de las grandes empresas familiares españolas. Presented at Proceedings from XIV Congreso ACEDE, Conocimiento y Competitividad, Murcia (Spain).

Sharma, P. (2008). Commentary: Familiness: Capital stocks and flowsbetween family and business. Entrepreneurship Theory and Practice, 32(6), 971-977.

Silva, R. y López de Ávila, A. (2007). El sector vitivinícola en España: El despertar de un gigante. Revista de Empresa, (22), 92-114.

Simon, D. y Hitt, M. (2003). Managing resources: Linking unique resources, management and wealth creation in family 
firms. Entrepreneurship Theory and Practice, 27(4), 339-358.

Suárez-Ortega, S. (2003). Export barriers: Insights from small and médium-sized firms, International Small Business Journal, 21(4), 403-419.

Swinth, R. y Vinton, K. (1993). Do familyowned businesses have a strategic advantage in international joint ventures? Family Business Review, 6(1), 19-30.

Tagiuri, R. y Davis, J. A. (1996). Bivalent attributtes of the family firm. Family Business Review, 9(2), 199-208.

Taylor, S.J. y Bogdan, R. (1992). Introducción a los métodos cualitativos de investigación: La búsqueda de significados. Barcelona: Paidos.

Terpstra, V. y Simonin, B.L. (1993). Strategic alliances in the Triad: An exploratory study. Journal of International Marketing, 1(1), 4-25.
Townsend, J. (2003). Understanding alliances: A review of international aspects in strategic marketing. Marketing Intelligence \& Planning, 21(3), 143-155.

Vallejo Martos , M.C. (2008). Is the Culture of Family Firms Really Different? A value-based model for its survival through generations. Journal of Business Ethics, 81(2), 261-279.

Varadarajan, R.P. y Cunningham, M.H. (1995). Strategic alliances: A synthesis of conceptual foundations. Journal of the Academy of Marketing Science, 23(4), 282-296.

Verón, E. (1996). La semiosis social: Fragmentos de una teoría de la discursividad. Barcelona: Gedisa.

d) Santander

